\title{
Melatonin and sleep in schizophrenia: a commentary
}

\section{Commentary}

Schizophrenia is a devastating mental illness with a worldwide prevalence of $1 \%$ with onset starting in late adolescence. It is characterized by a progressively deteriorating course with positive and negative symptoms including delusions, hallucinations, disorganized speech, grossly disorganized or catatonic behavior and negative symptoms. ${ }^{1}$ When patients with schizophrenia are acutely psychotic, sleep disturbances may range from some insomnia to no sleep. Severe insomnia is one of the prodromal symptoms of decompensation. Delayed sleep onset, maintenance insomnia and increased time awake is commonly seen in patients with schizophrenia regardless of their medication status. ${ }^{2,3}$ Early insomnia is highly characteristic of the disturbed sleep in schizophrenia. ${ }^{4}$ Reduced non-rapid eye movement, N3 sleep and REM sleep onset latency are disruptions in sleep architecture that have been noted in many studies. ${ }^{5}$

Sleep plays a significant role in the 24-hour circadian rhythm cycle which is a complex interaction between the sleep and wake promoting and inhibiting areas of the brain. The regulation of sleep and wakefulness involves a) the circadian system that intrinsically synchronizes the body functions to the light-dark cycle of the day, aligning activity to the light phase and sleep to the dark phase and b) the homeostatic component that builds up pressure for sleep during wakefulness and dissipates during sleep. ${ }^{5,6}$ The circadian rhythm is controlled by a set of CLOCK genes and their protein products. The transcription factors Circadian Locomotor Output Cycle Kaput(CLOCK) and Brain and Muscle Aryl hydrocarbon receptor nuclear translocator-like protein 1(BMAL1) activate a variety of genes including cytochrome (CRY1 and CRY2) and period (PER1, PER2 and PER3) genes. ${ }^{7}$ Disturbance in the circadian CLOCK, CRY1 and PER2 gene expression is seen both in newly diagnosed and chronic patients with schizophrenia who have very poor sleep. ${ }^{8}$ Melatonin (N-acetyl-5- methoxytrptamie) is one of the several neurotransmitters involved in induction and maintenance of sleep and it is synthesized in the pineal gland at night and follows a circadian rhythm driven by the hypothalamic suprachiasmatic nucleus where the timing apparatus is located. It acts through the $\mathrm{G}$ protein-coupled receptors known as MT1 (Me11a) and MT2 (Me11b). During the night, high levels of melatonin are present in the blood while low levels are found during the day. Disturbances in the diurnal cycle and low levels of melatonin during the night are associated with insomnia. Low melatonin levels and irregular patterns of its secretion have been seen in schizophrenic patients. ${ }^{9}$

Exogenous melatonin has been used to treat a variety of conditions that include sleep phase disorders such as jet lag and shift work. ${ }^{10}$ It has been used in treating primary sleep disorders and a meta-analysis ${ }^{11}$ concluded that melatonin had significant efficacy in reducing sleep latency, increasing total sleep time and improved overall sleep quality.

Melatonin has been studied both as a marker and as a therapeutic agent for treating schizophrenia. ${ }^{12}$ The results for using melatonin as a marker vary widely and are inconsistent. Melatonin as a therapeutic agent has been used in three studies to treat tar dive dyskinesia that showed higher dosed ameliorated some abnormal movements ${ }^{13-15}$ and detoxification from benzodiazepine dependence. ${ }^{16}$ There have been few studies done examining the usefulness of melatonin in treating
Volume 2 Issue 4 - 2018

\author{
Shabnam Sood \\ Assistant Clinical Professor, University of Arizona School of \\ Medicine-Phoenix, USA
}

\begin{abstract}
Correspondence: Shabnam Sood, Inpatient Medical Director Attending Psychiatrist, Maricopa Integrated Health System/ District Medical Group, Desert Vista Hospital, 570W Brown Road, Mesa, AZ 8520I, USA, Tel 48034420 03, Emailshabnam_sood@dmgaz.org
\end{abstract}

Received: April 23, 2018| Published: August 02, 2018

the insomnia in patients with schizophrenia. Electronic databases such as PUBMED and Google Scholar were used with the key wordsinsomnia, sleep, schizophrenia and melatonin and articles selected with relevance to using melatonin in treating insomnia in patients with schizophrenia.

In a randomized, double blinded crossover trial of 19 patients with schizophrenia; Shamir et al., ${ }^{17}$ demonstrated a beneficial effect of melatonin on improving sleep. Patients were treated with $2 \mathrm{mg}$ of extended release melatonin or placebo for 2 treatment periods of 3 weeks with a 1-week wash out period. All patients had low urinary outputs of melatonin and showed an improvement in sleep efficiency during the melatonin phase relative to the placebo phase. This was attributed to improvement in sleep initiation and/or maintenance. In another study, ${ }^{18}$ they used the first night effect (FNE) as a marker to explore neurobehavioral response of schizophrenic patients to melatonin. FNE is the tendency of individuals to sleep worse than normal during the first night of polysomnographic evaluation. 14 patients with schizophrenia were given $2 \mathrm{mg}$ of melatonin or placebo for 3 weeks with a wash out period of 1 week between treatments in a randomized, double-blinded crossover trial. Melatonin exaggerated the FNE in patients with schizophrenia with longer rapid eye movement sleep latency, lower sleep efficiency and lower duration of wakefulness during sleep. These were not seen in patients on placebo and suggest an improved ability of these patients to mobilize alertness in unfamiliar surroundings.

Melatonin given to stable schizophrenic outpatients with insomnia for at least weeks ${ }^{19}$ relative to control, showed significant improvements in the quality and depth of night-time sleep, reduced number of night-time awakenings and increased duration of sleep without drowsiness. Patients $(\mathrm{N}=40)$ were given flexibly dosed melatonin $(3-12 \mathrm{mg} / \mathrm{night})$ with a modal dose of $3 \mathrm{mg}$ or placebo. Decreased headache and dullness and improved functioning during the day were seen.

Alfonso et al., ${ }^{6}$ compared melatonin's sleep-promoting effect in 34 schizophrenic patients to healthy controls. They measured salivary levels of melatonin collected hourly for one night under dim light from 2000 to 2300 hours. Quality and patterns of sleep were measured using the Pittsburgh quality of Index score, sleep diary and wristactigraphy recordings for sleep-wake assessment and the Positive and Negative Symptom Scale used to assess psychopathology. The study showed that the endogenous melatonin sleep-promoting action 
seemed to be compromised in schizophrenic patients. The patients had worse quality of sleep, slept more at night but had poor sleep efficiency and melatonin levels correlated negatively with sleep latency and total sleep time.

Prolonged release melatonin was given to patients who were on long-term benzodiazepines and antipsychotic medications with diagnosis of schizophrenia, schizoaffective disorder or bipolar disorder. ${ }^{16}$ The patients were randomized to receive add on treatment with melatonin or placebo and benzodiazepines were tapered off gradually. Melatonin had no effect on sleep efficiency but patients had improved self-reported subjective sleep quality and these patients did not have rebound insomnia associated with benzodiazepine discontinuation.

Melatonin holds promise in treating insomnia in patients with schizophrenia but findings have been limited by only a few studies, which have a small sample size, lack of polysomnographic and objective measurements of sleep and low retention rates.

\section{Acknowledgments}

None

\section{Conflict of interest}

The author declared that there are no conflicts of interest.

\section{References}

1. American Psychiatric Association. Diagnostic and statistical manual of mental disorders. 5th ed. Arlington: American Psychiatric Publishing; 2013.

2. Tandon R, Shipley JE, Taylor S, et al. Electroencephalographic sleep abnormalities in schizophrenia. Relationship to positive/negative symptoms and prior neuroleptic treatment. Arch Gen Psychiatry. 1992;49(3):185-194.

3. Wulff K, Dijk DJ, Middleton B, et al. Sleep and circadian rhythm disruption in schizophrenia. Br J Psychiatry. 2012;200(4):308-316.

4. Benson KL. Sleep in schizophrenia: Impairments, Correlates, and Treatment. Psychiatr Clin of N Am. 2006;29(4):1033-1045.

5. Monti JM, Ba Hammam AS, Pandi Perumal SR, et al. Sleep and circadian rhythm deregulation in schizophrenia. Prog Neurosychopharmacol Biol Psychiatry. 2013;43:209-216.
6. Alfonso P, Figueira ML, Paiva T. Sleep promoting action of the endogenous melatonin in schizophrenia compared to healthy control. Int $J$ of Psychiatry in Clinical Practice. 2011;15(4):311-315.

7. Buhr ED, Takahashi JS. Molecular components of the Mammilian circadian clock. Handb Exp Pharmacol. 2013;(217):3-27.

8. Johansson AS, Owe-Larsson B, Hetta J, et al. Altered circadian clock gene expression in patients with schizophrenia. Schizophr Res. 2016;174(1-3):17-23.

9. Fanget F, Claustrat B, Dalery J, et al. Nocturnal plasma melatonin levels in schizophrenic patients. Biol Psychiatry. 1989;25(4):499-501.

10. Cardinali DP, Brusco LI, Lloret SP, et al. Melatonin in sleep disorders and jet lag. Neuro Endocrinol lett. 2002;23(Suppl 1):9-13.

11. Ferracioli-Oda E, Qawasmi A, Blocj MH. Meta-analysis: melatonin for treatment of primary sleep disorders. PLoS One. 2013;8(5):e63773.

12. Morera-Fumero AL, Abreu-Gonzalez P. Role of Melatonin in Schizophrenia. Int J Mol. Sci. 2013;14(5):9037-9050.

13. Shamir E, Barak Y, Shalman I, et al. Melatonin treatment for tardive dyskinesia: a double-blind, placebo-controlled, crossover study. Arch Gen Psychiatry. 2001;58(11):1049-1052.

14. Shamir E, Barak Y, Plopsky I, et al. Is melatonin treatment effective for tardive dyskinesia? J Clin Psychiatry. 2000;61(8):556-558.

15. Castro F, Carrizo E, Prieto De RD, et al. Effectiveness of melatonin in tardive dyskinesia. Invest Clin. 2011;52(3):252-260.

16. Baandrup L, Fagerlund B, Jennum P, et al. Prolonged-release melatonin versus placebo for benzodiazepine discontinuation in patients with schizophrenia: a randomized clinical trial-the SMART trial protocol. BMC Psychiatry. 2011;11:160.

17. Shamir E, Laudon M, Barak Y, et al. Melatonin improves sleep quality in patients with chronic schizophrenia. $J$ Clin Psychiatry. 2000;61(5):373-377.

18. Shamir E, Rotenberg VS, Laudon M, et al. First night effect of melatonin treatment in patients with chronic schizophrenia. J Clin Psychopharmacol. 2000;20(6):691-694.

19. Kumar SPN, Andrade C, Bhakta S, et al. Melatonin in schizophrenic outpatients with insomnia: a double blind, placebo-controlled study. $J$ Clin Psychitary. 2007;68(2):237-241. 